Aims: To evaluate immunohistochemical p16 expression and its prognostic utility in tongue SCC in two geographically and ethnically distinct populations.

Methods: 88 patients from the Sydney Head and Neck Cancer Institute and 44 patients from the National Cancer Centre Singapore with tongue SCC were identified from 2001 to 2012. p16 immunohistochemistry was performed. Strong nuclear and cytoplasmic staining in more than $70 \%$ of cells was interpreted as positive. Clinicopathologic data were obtained.

Results: p16 expression was seen in 18\% (16) of Australian and $23 \%$ (10) of Singaporean cases. The study populations showed no significant demographic differences. The Australian cohort had a significantly higher number of poorly differentiated cases ( $p=0.034$ ), however no significant differences were observed in other histopathological features such as depth of invasion, lymphovascular and perineural invasion, or pTNM staging. The majority of p16 positive cases in both cohorts were early pT stage tumours, with pN0 nodal status. p16 negative cases were more likely to receive post-operative radiotherapy in both cohorts. The interaction between p16 expression and institution was not significantly associated with overall survival.

Conclusion: Rates of p16 expression are similar between both study populations. The effect of p16 expression on overall survival did not vary according to the institution.

\section{PRIMARY SPLENIC MARGINAL ZONE LYMPHOMA: A CASE REPORT}

\section{Tanvi Shetty}

Melaka Manipal Medical College, Manipal, India

Background: Splenic marginal zone lymphoma (SMZL) is the term coined by Schmid et al. It accounts for less than $1 \%$ of all lymphomas. It is indolent B cell malignancy usually involving spleen, bone marrow and blood. It is made up of B cells that replace normal architecture of white pulp of spleen. Symptomatic splenomegaly is the presenting feature in almost all patients; anaemia related symptoms and B symptoms are rare. Spleen histology is the gold standard approach to establish a clear cut diagnosis of SMZL. A case of 65 year old male presenting only with splenomegaly and few atypical lymphoid cells in peripheral smear.

Aim: To determine the cause for massive splenomegaly.

Methods: Splenectomy was done and sections given from cut surface. They were stained with haematoxylin and eosin and microscopically analysed. Immunohistochemical (IHC) study was done for a panel of antibodies including CD5, CD10, CD20, bcl-2 and Cyclin D1.

Results: Microscopy showed effacement of the normal architecture, however there was a nodular architecture in the white pulp with small lymphocytes surrounded by medium to large lymphoid cells with dispersed chromatin and abundant pale cytoplasm which resembled the marginal zone cells. IHC study showed positivity for CD20 and bcl-2. It was negative for CD5, CD10 and Cyclin D1. Considering all the findings a diagnosis of splenic marginal zone lymphoma (SMZL) was made.

Conclusion: In this particular case, clinically lymphoma was not suspected, as splenomegaly was the only finding. Patient was thoroughly investigated for various causes of splenomegaly but only histopathological evaluation of spleen along with IHC study was diagnostic.

\section{EXPRESSION OF HYPOXIA-INDUCIBLE FACTOR- 1A SUBUNIT IS PARTLY CONTROLLED BY UPREGULATION OF UBIQUITIN CARBOXYL- TERMINAL ESTERASE L3}

Yonghong Shi, Jing Zhao, Ling Hai

Department of Pathology, Inner Mongolia Medical University, Huhhot City, PR China

Our previous study found that triple-negative breast cancer line MDA-MB-231 showed a higher expression of hypoxia-inducible factor $1 \alpha$ (HIF-1 $\alpha$ ) under nomoxic condition and a higher capability of cell growth and invasive/migratory than other breast cancer cell lines. SiRNA targed HIF- $1 \alpha$ interrupted HIF$1 \alpha$ expression and suppressed the ability of cell growth and invasion/migration with a downregulation of MMP8/17/10/13 mRNA in MDA-MB-231 cell. Ubiquitin carboxyl-terminal hydrolases L3 (UCH-L3) is a kind of deubiquitinating enzyme which may hydrolyze the ubiquitinated proteins from polyubinquited compounds before degradation by proteolytic ezyme system and release ubiquitin for recycle. HIF- $1 \alpha$ is a regulatory subunit by oxygen and degraded by ubiquitin-proteasome pathway. This study was aimed to probe the role of UCH-L3 in modulation of HIF- $1 \alpha$ expression and its effect on malignant behavior as well as the mechanism involved.

Methods: siRNA was used to interfere HIF-1 $\alpha$ or UCH-L3 expression. Bidirectional gel electrophoresis and mass spectormetry were used to explore differential proteins by comparing the control group with HIF-1 $\alpha$ targeted siRNA group. Real time PCR and western blot were used respectively to detect mRNA expression and protein Cell count, flow cytometry and Hoechst staining were used to evaluate cell growth and apoptosis. Matrigel invasion and wound scratch assay were performed to measure the ability of cell invasion and migration.

Results: Protein expression of UCH-L3 in MDA-MB-231 cells transfected with HIF-1 $\alpha$-targeted siRNA was detected by Bidirectional gel electrophoresis and mass spectormetry. Constitutive expression of UCH-L3 by transfected cells with expression plasmid resulted in a decrease of HIF- $1 \alpha$ mRNA expression accompanied a disability of migration/invasion. SiRNA targeted UCH-L3 increase protein expression of HIF- $1 \alpha$ to some extent and facilitated clone formation and accelerated cell growth. Further constitutively lentiviral vector of UCH-L3 was transfected into cells resulting in a significant reduction of HIF-1 $\alpha$ protein expression and a decrease of cell apoptosis. While addition of protease inhibitor MG231 in those cells arrested reduction of HIF-1 $\alpha$ protein.

Conclusion: UCH-L3 may be an important factor in regulating degradation of HIF- $1 \alpha$ protein and regressing malignant effect of HIF-1 on tumor. Constitutive expression of UCH-L3 or application of synthetic analogues could probably be a potential means for the treatment of triple-negative breast cancer.

\section{AN INTEROBSERVER REPRODUCIBILITY ANALYSIS OF KI67 VISUAL ASSESSMENT IN BREAST CANCER}

Ruohong Shui, Baohua Yu, Rui Bi, Fei Yang, Wentao Yang Department of Pathology, Fudan University Shanghai Cancer Center, Department of Oncology, Shanghai Medical College, Fudan University, Shanghai, China 